\title{
Clustering of European Climates and Representative Climate Identification for Building Energy Simulation Analyses
}

\author{
Giovanni Pernigotto ${ }^{1}$, Angélica Walsh ${ }^{2,3}$, Andrea Gasparella ${ }^{1}$, Jan L.M. Hensen ${ }^{3}$ \\ ${ }^{1}$ Free University of Bozen-Bolzano, Bolzano, Italy \\ ${ }^{2}$ UNICAMP State University of Campinas, Brazil \\ ${ }^{3}$ Eindhoven University of Technology, Eindhoven, The Netherlands
}

\begin{abstract}
This research presents a methodology to cluster weather climates with the purpose of generating homogeneous and distinct groups suitable for the selection of representative cities, to use as reference for robust generalization of building simulation findings. The proposed methodology is based on Hierarchical Clustering and KolmogorovSmirnov tests, applied to dry bulb temperature, water vapour partial pressure and solar irradiation data. As an example, a dataset with more than 300 European climates is analysed, a new classification is prepared and compared with the well-known Köppen-Geiger system. Results on this paper provide preliminary indications of the large potential of this approach to identify representative cities for Building Energy Simulation analyses.
\end{abstract}

\section{Introduction}

The preparation and the selection of weather files for Building Energy Simulation, $B E S$, are particularly important to ensure representative and robust $B E S$ findings. The literature is rich of contributions about typical, future or extreme reference years as reported by Pernigotto et al. (2014). However, when it comes to identify a location as representative for an entire region, there is no reference procedure to adopt. The choice is often arbitrary and made by researchers based on personal expertise and non-climatic concerns (Walsh et al., 2017).

In some cases, selection of representative climates relies on well-known climate classifications, such as the Köppen-Geiger system (Peel et al., 2007), which, however, was developed for purposes different than $B E S$ analysis. In other cases, national guidelines, such as the ANSI/ASHRAE 169 (ASHRAE, 2013) can be followed but, often, this type of climate classification is based only on heating and cooling degree-days scales and precipitation values. This means that weather variables such as humidity and solar irradiance are neglected, even though they may have a huge impact on latent and sensible air heat balances in building ventilation analysis, or on solar gains, especially in highly glazed buildings, or for solar assisted HVAC systems, etc.

In Europe, most countries adopted climatic zoning for building energy efficiency applications (Walsh et al., 2017): some relied on weather-based approaches (Pernigotto and Gasparella, 2018) while others on performance-based approaches (Walsh et al., 2018). In spite of that, there is a lack of information regarding a climate classification for building energy efficiency applications for the whole continent, which would be particularly relevant for the development of common building energy policies at European level. Moreover, the selection of representative cities could facilitate also the definition of energy policies at regional or international level when high-resolution data are not required.

After a critical discussion of the Köppen-Geiger climate classification system, we proposed a new weather-based methodology based on statistical and data-mining techniques (Hierarchical Clustering and KolmogorovSmirnov tests) to study a dataset of climates and select the most representative ones for $B E S$ analyses The new approach was applied to the European context to cluster a large sample of more than 300 typical reference years collected from the EnergyPlus weather database, to define homogeneous non-overlapping zones based on the most important weather variables for BES (i.e., dry bulb temperature, water vapour partial pressure, and global horizontal solar irradiation).

\section{Methods}

\section{Dataset of climates}

As a first step, the climates available from EnergyPlus online database (https://energyplus.net/weather) were analysed to define the dataset for the study. The following criteria were adopted: (1) only European countries and territories were considered; (2) in case of more weather files available for the same localities, IWEC (International Weather for Energy Calculations) were preferred. In the case of Italy, since the online data belong to the IGDG series, developed from the weather data collected in the period 1951-1970 and now outdated, we used the reference years recently developed and published by the Italian CTI (Comitato Termotecnico Italiano, https://try.cti2000.it/) and coherent with the current Italian technical standard UNI 10349-1 (2016).

For each climate, the variables more relevant for the building energy performance, i.e., air temperature, humidity and solar radiation, were studied. In order to express the humidity independently of the temperature, the water vapour partial pressure $W V P$ was considered instead of relative humidity $R H$. As a whole, each climate was characterized by means of its monthly averages of dry bulb temperature $D B T$ and water vapour partial pressure $W V P$ and the monthly integral of global horizontal irradiation GHI. Wind speed data, instead, were neglected because in many cases are representative only of local 
conditions. For each variable and location, the average of the 12 monthly values and their spread (namely, annual averages and spreads) were used as statistics in the following steps of the research.

\section{Assessment of the Köppen-Geiger classification}

Considering that one of the targets of this research is to provide homogenous and distinct groups of climatic classes for Europe, from which identifying some reference localities, the set of climates included in the study was split according to the current Köppen-Geiger classification. Homogeneity and overlapping were evaluated by means respectively of the standard deviations and the mean values of the annual averages and spreads of $D B T, W V P$ and $G H I$, as shown in Figure 1.

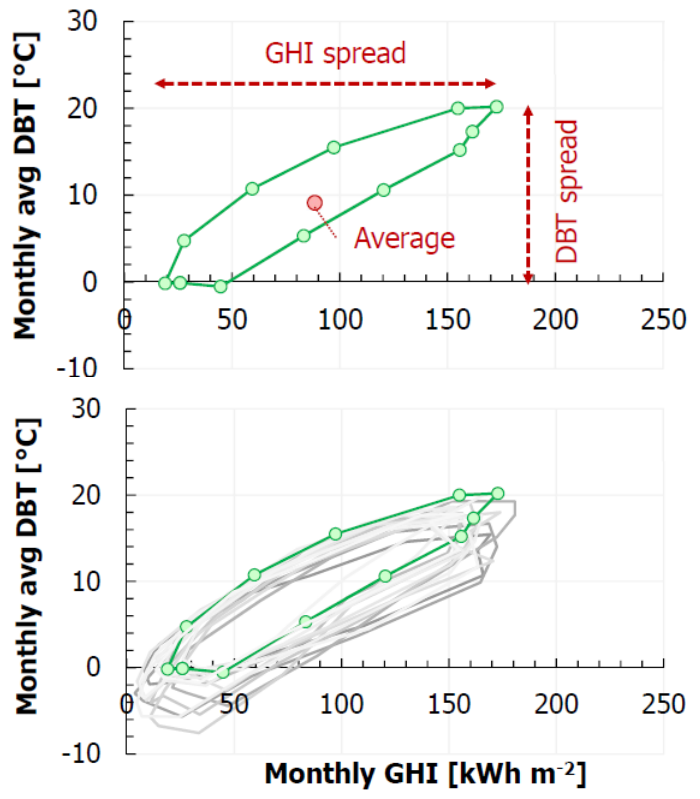

Figure 1: Definition of annual statistics for the weather variables characterizing a locality - average and spread (top); homogeneity of a class of climates (bottom).

\section{New classifications and representative locations}

Following the methodology defined in a previous work (Pernigotto and Gasparella, 2018), Hierarchical Clustering on normalized weather statistics (i.e., annual averages and spreads) were adopted. Hierarchical Clustering, specifically, was chosen for its ability to identify outliers without a preselected number of clusters as input. First, each weather variable was studied separately in dedicated clusterings and then combined in a global clustering considering all of them. Indeed, differently from the preliminary studies, in this research the dataset of more than 300 climates was considered large enough to allow for meaningful results of global clustering, without any hierarchy between the variables and necessity of sub-clustering. Geographical distances were not considered in clustering and, thus, uneven distributions of localities did not affect the classification. For each step, obtained climate classes were analysed in terms of overlapping and homogeneity as described in the previous section about the Köppen-Geiger classification. In particular, each new grouping solution was compared to the original Köppen-Geiger ones. The selection of the representative climates for each climatic zone relied on Kolmogorov-Smirnov tests. For each new climatic class and each variable, monthly series were used for the definition of an annual reference profile, taken as reference for Kolmogorov-Smirnov tests. Similarly to the approach used for the definition of a typical year according to EN ISO 15927-4 (CEN, 2005; Pernigotto et $a l ., 2014)$, representativeness of each city for $D B T, W V P$ and $G H I$ was assessed independently and, for each, a partial ranking was built. Finally, a global ranking was prepared for each climatic zone and a representative city identified.

\section{Results and discussion}

\section{Dataset of climates}

The dataset of climates includes 318 different localities. The climates are not evenly distributed on the European territory: most of them are in Italy (110 localities), in Poland (61) and in Spain (46). As regards the distribution in the different Köppen-Geiger classes, an overview can be seen in Figure 2 and in Table 1. The considered localities are distributed across 11 Köppen-Geiger classes but those with at least 10 locations are only 7: BSk (arid cold steppe climate, in Spain and Turkey), Csa (temperate climate with dry and hot summer in the Mediterranean areas), Csb (temperate climate with dry and warm summer, in the southern France and in the western Iberian peninsula), Cfa (temperate climate without dry season and with hot summer, in the north of Italy and in the Balkans), Cfb (temperate climate without dry season and with warm summer, in the western Europe), Dfb (cold climate without dry season and with warm summer, in the central and eastern Europe), Dfc (cold climate without dry season and with cold summer, in Scandinavia and in the north of Russia). Dfb, Csa and Cfb are the three most populated climate classes, representing respectively $29.2 \%, 23.6 \%$ and $17.9 \%$ of the dataset.

\section{Assessment of the Köppen-Geiger classification}

The Köppen-Geiger classes including at least 10 items (i.e., $97.8 \%$ of the sample) were analysed according to the approach explained in the methods. For each weather variable and class, annual mean values and standard deviations were calculated and represented in Figure 3.

Considering the annual $D B T$, mean annual averages range from $4.1{ }^{\circ} \mathrm{C}$ (Dfc) to $15.8{ }^{\circ} \mathrm{C}$ (Csa), with very similar mean spreads around $20{ }^{\circ} \mathrm{C}$ for $\mathbf{C f a}$, Dfb and Dfc and between 14.4 and $17.2{ }^{\circ} \mathrm{C}$ for the other classes. Some overlapping can be observed, in particular between the mean annual averages of $\mathbf{C s b}$ and $\mathbf{C f a}$ classes (around $13{ }^{\circ} \mathrm{C}$ ) and of BSk and Csa classes (around $15.5^{\circ} \mathrm{C}$ ). Moreover, while the Csb and Cfa can be clearly distinguished by two different mean annual spreads of monthly mean temperatures, the same is not true for BSk and Csa for the current dataset, which are almost overlapped in Figure 3. Considering the homogeneity of the classes for $D B T$, standard deviations of annual averages are within or close to $2{ }^{\circ} \mathrm{C}$ for all classes except Dfc. Regarding the standard deviations of annual spreads, instead, only Cfa, Csa, BSk and Dfb are close or within 
$2{ }^{\circ} \mathrm{C}$, Cfb and $\mathbf{C s b}$ between 3 and $4{ }^{\circ} \mathrm{C}$ and Dfc almost $5{ }^{\circ} \mathrm{C}$. Analysing $D B T$, it can be concluded that some classes (representing about $47 \%$ of the sample) are overlapped in terms of annual averages and annual spreads and in around $25 \%$ of the sample there is poor homogeneity.

Focusing on the $W V P$, mean annual averages range from $719 \mathrm{~Pa}$ (Dfc) to $1284 \mathrm{~Pa}$ (Csa), with mean spreads between $642 \mathrm{~Pa}$ (Csb) and $1276 \mathrm{~Pa}$ (Cfa). As far as humidity is concerned, overlapping is less marked than for dry bulb temperature. Indeed, only $\mathbf{C f b}$ and $\mathbf{C s b}$ have very close mean annual averages (around $1040 \mathrm{~Pa}$ ) but different mean spread (respectively, $870 \mathrm{~Pa}$ and $642 \mathrm{~Pa}$ ). The homogeneity, on the contrary, is much poorer: only Cfa and Dfb have standard deviations of the annual averages within $10 \%$ and Csb and Dfc values are even larger than $20 \%$. Except for Cfa and Dfb, the standard deviations of the spread are between 20 and $30 \%$ for all classes. As expected, since the water vapour partial pressure is not considered in Köppen-Geiger classification, homogeneity in climate zones cannot be taken for granted. In particular, in two classes, Csb and Dfc, dissimilarities are high, even if this affects only $7.2 \%$ of the sample.

Finally, for the GHI mean annual averages, ranges between $77 \mathrm{kWh} \mathrm{m}^{-2}$ (Dfc) and $133.9 \mathrm{kWh} \mathrm{m}^{-2}$ (BSk) can be found. The mean annual spreads can be distinguished into two groups: $\mathbf{C f b}, \mathbf{D f b}$ and $\mathbf{D f c}$ around $140 \mathrm{kWh} \mathrm{m}^{-2}$

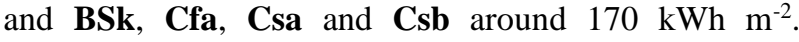
Overlapping issues can be recorded for classes BSk, Csa and Csb, with very similar mean values of both annual averages and spreads. The homogeneity is generally good for both averages and spreads in case of BSk, Cfa, Csa and Csb, which are within or close to $10 \%$ of standard deviations for both parameters. A different situation is recorded for $\mathbf{C f b}$, Dfb and Dfc (i.e., more than half of the sample), which have a standard deviation of the annual averages between $15 \%$ and $20 \%$ and a standard deviation of annual spreads between $10 \%$ and $20 \%$.

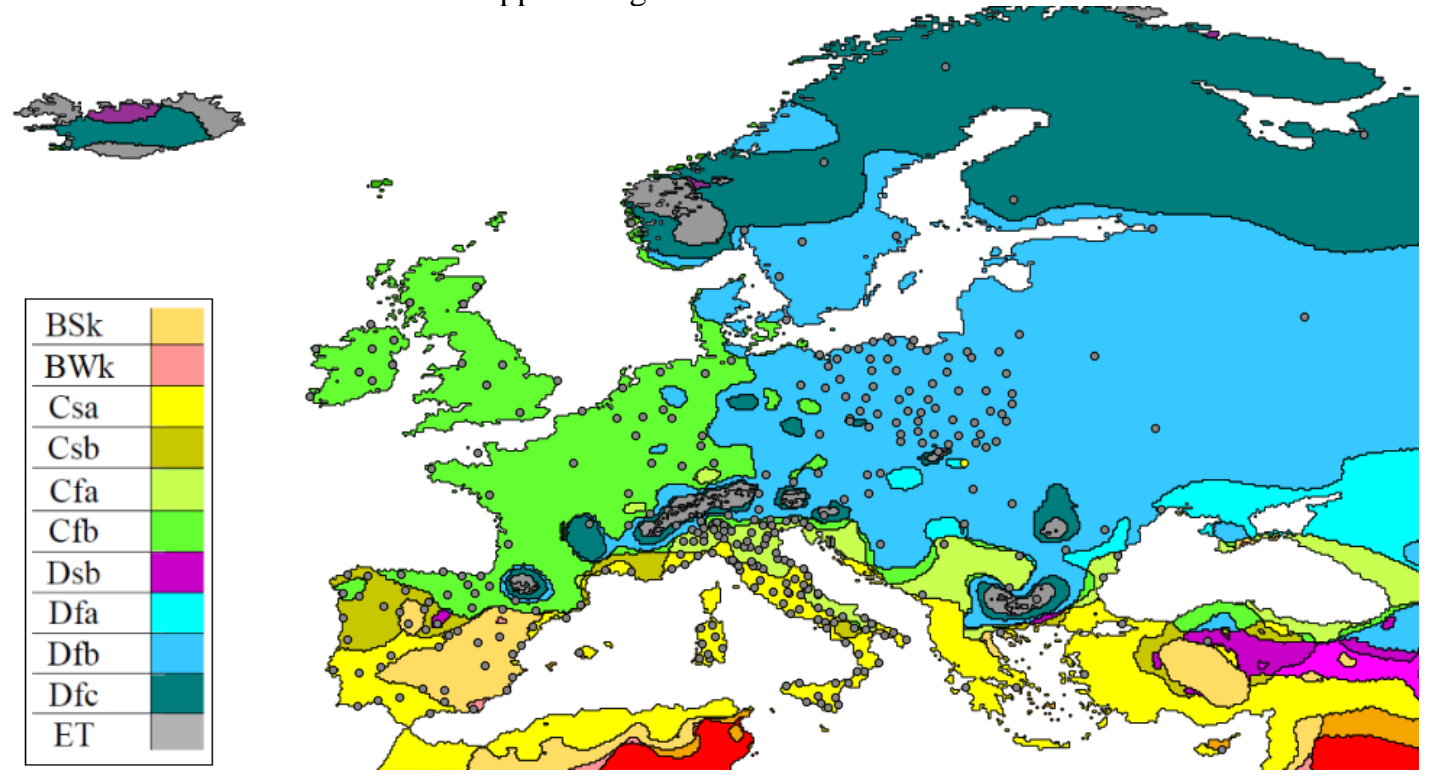

Figure 2: European climate zones according to Köppen-Geiger (main classes in the map legend). Grey dots indicate the localities included in the study. Map prepared with QGIS v. 3.4.2 based on the Köppen-Geiger GIS climate map by NASA ORNL DAAC.

Table 1: Considered climates and Köppen-Geiger classes. In grey classes with a number of entries larger than 10.

\begin{tabular}{|c|c|c|c|}
\hline Köppen-Geiger class & Description & Number of cities & Fraction of cities \\
\hline BSk & Arid cold steppe climate & 14 & $4.4 \%$ \\
\hline BWk & Arid desert cold climate & 1 & $0.3 \%$ \\
\hline Csa & Temperate climate with dry and hot summer & 75 & $23.6 \%$ \\
\hline Csb & Temperate climate with dry and warm summer & 12 & $3.8 \%$ \\
\hline Cfa & Temperate climate without dry season and with hot summer & 49 & $15.4 \%$ \\
\hline $\mathbf{C f b}$ & Temperate climate without dry season and with warm summer & 57 & $17.9 \%$ \\
\hline Dsb & Cold climate with dry and warm summer & 2 & $0.6 \%$ \\
\hline Dfa & Cold climate without dry season and with hot summer & 3 & $0.9 \%$ \\
\hline Dfb & Cold climate without dry season and with warm summer & 93 & $29.2 \%$ \\
\hline Dfc & Cold climate without dry season and with cold summer & 11 & $3.5 \%$ \\
\hline \multirow[t]{2}{*}{ ET } & Polar and tundra climate & 1 & $0.3 \%$ \\
\hline & & 318 & $100 \%$ \\
\hline
\end{tabular}



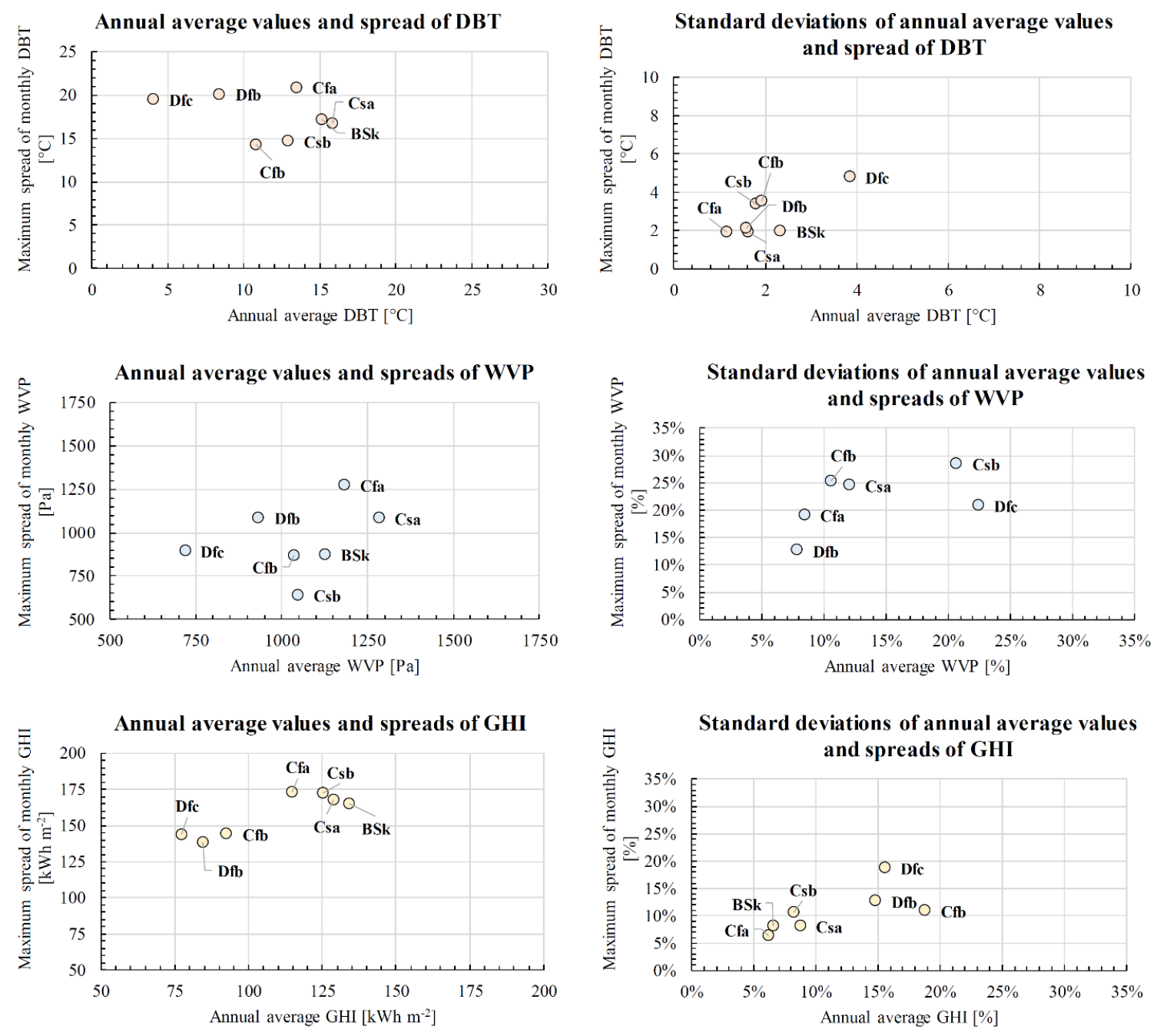

Figure 3: Mean values (left) and standard deviations (right) of annual averages and spreads of dry bulb temperature $(D B T)$, water vapour partial pressure (WVP) and global horizontal irradiation (GHI) for Köppen-Geiger classes.

\section{Partial clusterings}

A first set of clustering was performed considering each weather variable at a time, coupling both statistics (i.e., normalized annual average and spread). As shown in Figure 4, it is possible to observe that in all three clustering 7 clusters can be identified by cutting the dendrogram at a height around 0.5 . In the case of $D B T, 2$ clusters out of 7 have a number of elements lower than 10 while in $W V P$ and $G H I$ this is true for only a cluster. Clearly, the cutting level in the dendrogram is an arbitrary choice which has to balance the number of desired clusters and their homogeneity: the higher height is considered, the lower the number of classes but also the lower their homogeneity. Mean values and standard deviations of annual statistics are reported in Table 2.

Regarding the $D B T$ clustering, the obtained 7 classes have a better distribution than the original Köppen-Geiger ones for the dry bulb temperature, without overlapping and with increased homogeneity (i.e., standard deviation lower or around $2{ }^{\circ} \mathrm{C}$ ) except for classes $\mathbf{1}^{\mathrm{DBT}}$ and $2^{\mathrm{DBT}}$, which include just 4 items each. Improvements can be observed also for $W V P$ mean annual values and spreads but followed by larger standard deviations. Overlapping issues for $G H I$ are still present and standard deviations do not show significant variations.

In the $W V P$ clustering, mean annual values have the best improvement for humidity, as expected, but also $D B T$ shows absence of overlapping. In the case of $G H I$, the classes show very close annual averages with approximately the same spread, ranging between 140 and $170 \mathrm{kWh} \mathrm{m}^{-2}$, similarly to the original classification. Standard deviations for WVP annual averages are within $10 \%$ except for class $\mathbf{1}^{\mathbf{W V P}}$ and standard deviations for $W V P$ annual spreads are within or close to $15 \%$ except for class $\mathbf{2}^{\mathbf{W V P}}$, highlighting a significant improvement in uniformity with respect to Köppen-Geiger classification. No significant changes are detected for $D B T$ standard deviations and a light worsening for the $G H I$ ones.

Finally, in the GHI clustering, even if overlapping issues are fixed for the solar irradiation, it is not for dry bulb temperature and water vapour partial pressure. Homogeneity is largely improved for GHI, with standard deviations lower than $10 \%$ for annual values and within or close to $5 \%$ for the spreads. Nevertheless, uniformity 
and standard deviations are slightly worsened for $D B T$ and significantly worsened for $W V P$.

In conclusion of this section, partial clustering succeeded in solving overlapping and uniformity issues for the selected weather variable. However, considering one variable at a time is insufficient to ensure a global improvement with respect to the Köppen-Geiger system.

\section{Global clustering}

After considering the outcome of the partial clustering, all variables were included in a global clustering, whose results are reported in Figures 5 - 7. As it can be noticed in Figure 5, the inclusion of all the weather variables makes more difficult to find homogeneous groups and consequently the vertical axis describes a slightly wider range. In this framework, the proper cutting level to define the clusters was set around 1, generating 7 classes with only 1 with just 2 items.
Excluding class 1 (with just 2 climates) and analysing the mean values and the standard deviations of annual averages and spreads of $D B T, W V P$ and $G H I$, improvements were recorded with respect to the original Köppen-Geiger classification. Considering $D B T$, there is limited overlapping - even if classes $\mathbf{3}$ and $\mathbf{4}$ and classes 5 and $\mathbf{6}$ present, respectively, similar mean annual averages, they show different mean spreads. The standard deviation of average $D B T$ is within or close to $2{ }^{\circ} \mathrm{C}$ for all classes and the same holds also for the standard deviation of $D B T$ spread except for classes 2 and 4. Regarding WVP, all classes are characterized by well-distinguished averages and spreads and increased homogeneity: the standard deviation of average $W V P$ is within $15 \%$ and the one for the spread between $10 \%$ and $25 \%$. Finally, the uniformity of GHI in the developed classes is significantly improved (i.e., generally within $10 \%$ standard deviation except for class 3 ) but there are still overlapping issues (e.g., between classes $\mathbf{3}$ and $\mathbf{4}$ ).

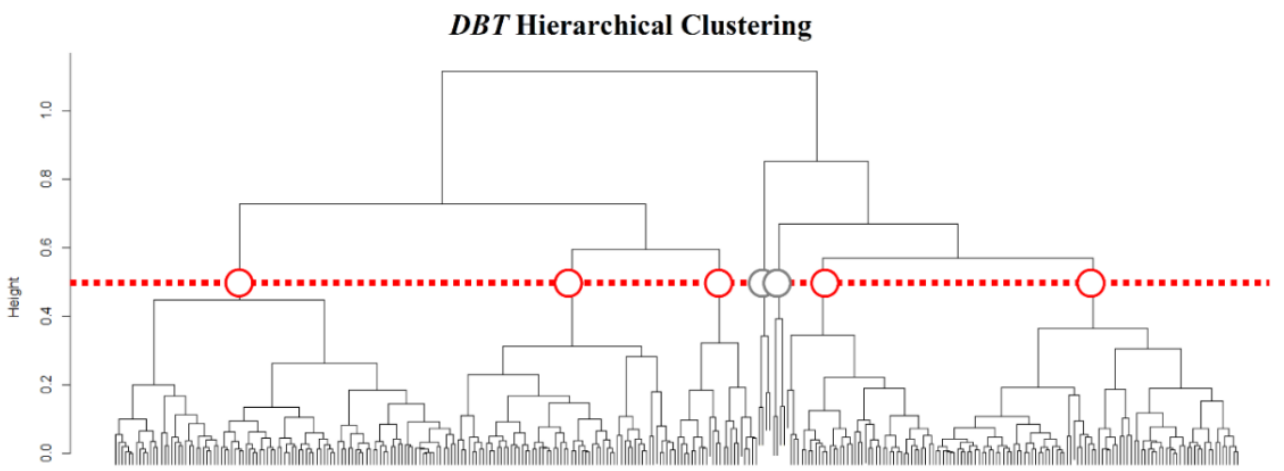

WVP Hierarchical Clustering

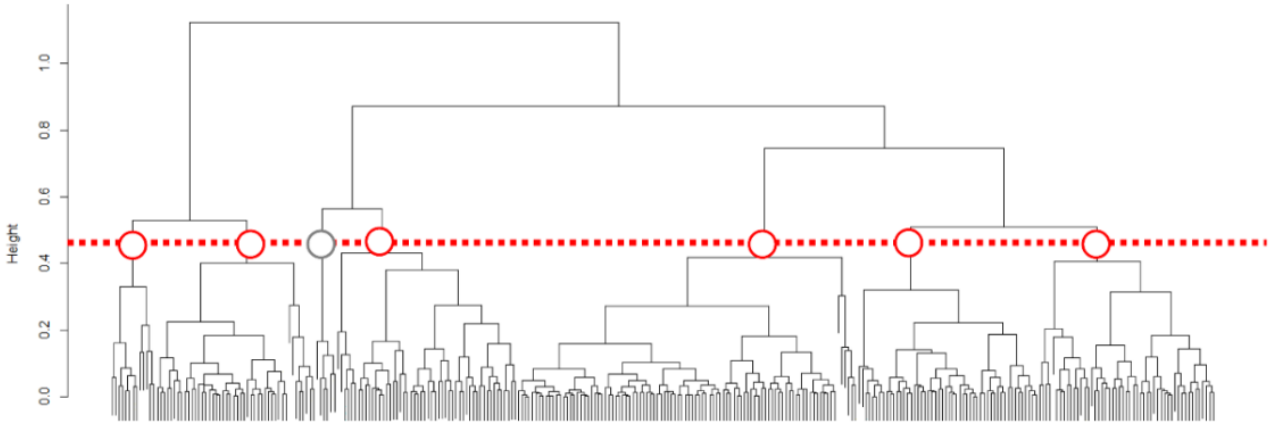

GHI Hierarchical Clustering

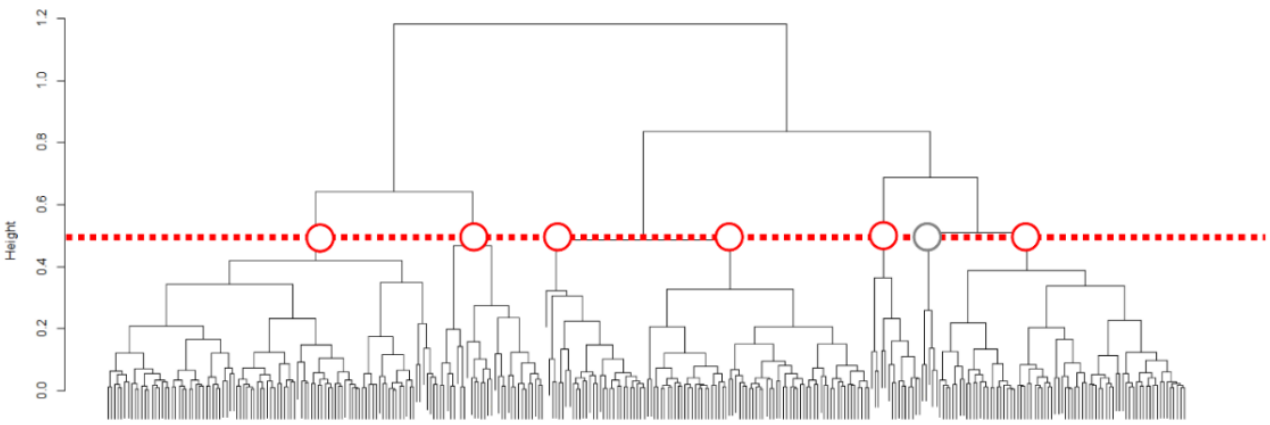

Figure 4: Partial hierarchical clustering with DBT, WVP and GHI statistics. The red line represents the height chosen for determining the number of clusters, identified with the circles (red if included climates are at least 10, grey if lower). 
Table 2: Partial hierarchical clustering with DBT, WVP and GHI: mean values and standard deviations for each class and weather statistics (i.e., annual averages and spreads).

\begin{tabular}{|c|c|c|c|c|c|c|c|c|}
\hline \multicolumn{9}{|c|}{ DBT Hierarchical Clustering } \\
\hline & & \multicolumn{7}{|c|}{ Classes and fraction of sample } \\
\hline & Quantities & $1(1.3 \%)$ & $2(1.3 \%)$ & $3(13.2 \%)$ & $4(27 \%)$ & $5(7.2 \%)$ & $6(30.5 \%)$ & $7(19.5 \%)$ \\
\hline \multirow{3}{*}{$\begin{array}{l}\text { Annual } \\
\text { Average }\end{array}$} & $D B T\left[{ }^{\circ} \mathrm{C}\right]$ & $2.6 \pm 3.0$ & $3.1 \pm 3.4$ & $7.6 \pm 1.5$ & $9.5 \pm 1.7$ & $11.1 \pm 2.1$ & $13.5 \pm 1.7$ & $16.4 \pm 1.4$ \\
\hline & $W V P[\mathrm{~Pa}]$ & $690 \pm 21 \%$ & $672 \pm 19 \%$ & $894 \pm 9 \%$ & $952 \pm 9 \%$ & $1087 \pm 11 \%$ & $1142 \pm 11 \%$ & $1334 \pm 10 \%$ \\
\hline & $G H I\left[\mathrm{kWh} \mathrm{m}^{-2}\right]$ & $76 \pm 18 \%$ & $67 \pm 8 \%$ & $83 \pm 12 \%$ & $91 \pm 21 \%$ & $89 \pm 19 \%$ & $118 \pm 9 \%$ & $130 \pm 9 \%$ \\
\hline \multirow{3}{*}{$\begin{array}{l}\text { Annual } \\
\text { Spread }\end{array}$} & $\mathrm{DBT}\left[{ }^{\circ} \mathrm{C}\right]$ & $27.7 \pm 2.0$ & $13.8 \pm 1.8$ & $21.2 \pm 1.1$ & $17.5 \pm 1.6$ & $10.4 \pm 1.1$ & $20.6 \pm 1.9$ & $15.6 \pm 1.4$ \\
\hline & $W V P[\mathrm{~Pa}]$ & $1203 \pm 24 \%$ & $675 \pm 9 \%$ & $1098 \pm 9 \%$ & $914 \pm 22 \%$ & $745 \pm 18 \%$ & $1182 \pm 24 \%$ & $1097 \pm 24 \%$ \\
\hline & $G H I\left[\mathrm{kWh} \mathrm{m}^{-2}\right]$ & $160 \pm 5 \%$ & $119 \pm 19 \%$ & $142 \pm 12 \%$ & $144 \pm 15 \%$ & $136 \pm 11 \%$ & $172 \pm 8 \%$ & $161 \pm 8 \%$ \\
\hline \multicolumn{9}{|c|}{ WVP Hierarchical Clustering } \\
\hline & & \multicolumn{7}{|c|}{ Classes and fraction of sample } \\
\hline & Quantities & $1(4.1 \%)$ & $2(14.5 \%)$ & $3(30.8 \%)$ & $4(16.4 \%)$ & $5(16.4 \%)$ & $6(16 \%)$ & $7(1.9 \%)$ \\
\hline \multirow{3}{*}{$\begin{array}{l}\text { Annual } \\
\text { Average }\end{array}$} & $D B T\left[{ }^{\circ} \mathrm{C}\right]$ & $4.8 \pm 3.5$ & $10.9 \pm 2.2$ & $8.7 \pm 2.0$ & $13.1 \pm 2.3$ & $13.8 \pm 1.8$ & $16 \pm 1.7$ & $17.4 \pm 1.1$ \\
\hline & $W V P[\mathrm{~Pa}]$ & $707 \pm 15 \%$ & $939 \pm 8 \%$ & $943 \pm 8 \%$ & $1105 \pm 7 \%$ & $1252 \pm 8 \%$ & $1306 \pm 7 \%$ & $1574 \pm 4 \%$ \\
\hline & $G H I\left[\mathrm{kWh} \mathrm{m}^{-2}\right]$ & $81 \pm 22 \%$ & $104 \pm 26 \%$ & $87 \pm 16 \%$ & $110 \pm 19 \%$ & $115 \pm 8 \%$ & $129 \pm 10 \%$ & $134 \pm 9 \%$ \\
\hline \multirow{3}{*}{$\begin{array}{l}\text { Annual } \\
\text { Spread }\end{array}$} & $\operatorname{DBT}\left[{ }^{\circ} \mathrm{C}\right]$ & $19.7 \pm 4.1$ & $15.2 \pm 3.3$ & $20.3 \pm 2.3$ & $16.7 \pm 3.6$ & $19.6 \pm 2.8$ & $16.3 \pm 2.9$ & $16.0 \pm 0.5$ \\
\hline & $W V P[\mathrm{~Pa}]$ & $855 \pm 16 \%$ & $661 \pm 20 \%$ & $1098 \pm 10 \%$ & $875 \pm 14 \%$ & $1444 \pm 9 \%$ & $1057 \pm 14 \%$ & $1398 \pm 5 \%$ \\
\hline & $G H I\left[\mathrm{kWh} \mathrm{m}^{-2}\right]$ & $148 \pm 18 \%$ & $156 \pm 14 \%$ & $142 \pm 14 \%$ & $159 \pm 14 \%$ & $165 \pm 8 \%$ & $165 \pm 10 \%$ & $163 \pm 6 \%$ \\
\hline \multicolumn{9}{|c|}{ GHI Hierarchical Clustering } \\
\hline & & \multicolumn{7}{|c|}{ Classes and fraction of sample } \\
\hline & Quantities & $1(9.4 \%)$ & $2(4.7 \%)$ & $3(20.8 \%)$ & $4(23 \%)$ & $5(1.6 \%)$ & $6(30.8 \%)$ & $7(9.7 \%)$ \\
\hline \multirow{3}{*}{$\begin{array}{l}\text { Annual } \\
\text { Average }\end{array}$} & $D B T\left[{ }^{\circ} \mathrm{C}\right]$ & $7.7 \pm 2.6$ & $5.1 \pm 2.5$ & $8.6 \pm 1.2$ & $12.2 \pm 2.4$ & $13.8 \pm 1.2$ & $14 \pm 1.8$ & $17 \pm 1.5$ \\
\hline & $W V P[\mathrm{~Pa}]$ & $915 \pm 13 \%$ & $772 \pm 15 \%$ & $954 \pm 7 \%$ & $1122 \pm 14 \%$ & $1216 \pm 15 \%$ & $1149 \pm 15 \%$ & $1316 \pm 13 \%$ \\
\hline & $G H I\left[\mathrm{kWh} \mathrm{m}^{-2}\right]$ & $75 \pm 9 \%$ & $78 \pm 10 \%$ & $81 \pm 7 \%$ & $106 \pm 9 \%$ & $109 \pm 9 \%$ & $123 \pm 6 \%$ & $142 \pm 4 \%$ \\
\hline \multirow{3}{*}{$\begin{array}{l}\text { Annual } \\
\text { Spread }\end{array}$} & $D B T\left[{ }^{\circ} \mathrm{C}\right]$ & $18.1 \pm 2.5$ & $22.3 \pm 4.0$ & $16.8 \pm 4.2$ & $18.8 \pm 3.3$ & $15.5 \pm 5$ & $18.7 \pm 2.8$ & $15.9 \pm 2$ \\
\hline & $W V P[\mathrm{~Pa}]$ & $979 \pm 10 \%$ & $1043 \pm 18 \%$ & $946 \pm 21 \%$ & $1165 \pm 24 \%$ & $1218 \pm 12 \%$ & $1049 \pm 30 \%$ & $989 \pm 29 \%$ \\
\hline & $G H I\left[\mathrm{kWh} \mathrm{m}^{-2}\right]$ & $116 \pm 6 \%$ & $161 \pm 4 \%$ & $135 \pm 4 \%$ & $156 \pm 6 \%$ & $127 \pm 4 \%$ & $178 \pm 5 \%$ & $160 \pm 6 \%$ \\
\hline
\end{tabular}

\section{Global Hierarchical Clustering}

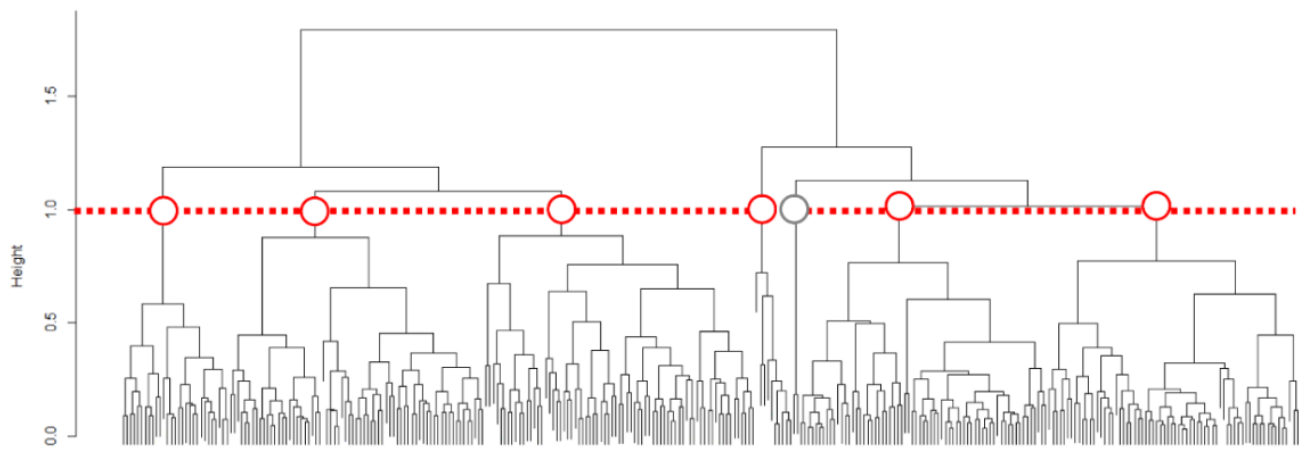

Figure 5: Global hierarchical clustering. The light red line represents the height chosen for determining the number of clusters, identified with the circles (red if included climates are at least 10, grey if lower).

Considering geographical distribution of the generated classes (Figure 7):

- Class 1 (grey) includes just two alpine climates (Kasprowy Wierch and Sniezka), at the border between Poland and Czech Republic;

- Class 2 (yellow) includes only 10 climates in Scandinavia and Russia;

- Class 3 (blue) is composed by 68 localities (i.e., about $21 \%$ of the sample), distributed in eastern Europe and in the Alpine region or mountain areas in the Balkans;
- Class 4 (green) has 67 localities (i.e., about $21 \%$ of the sample), mainly in western Europe and in part of Poland;

- Class 5 (purple) has 69 localities (i.e., $22 \%$ ), in the centre and north of Italy and in the north of the Balkans;

- Class 6 (light blue) includes 29 cities (i.e., $9 \%$ ), mostly in Spain with a few localities in the mountain regions of the south of Italy;

- Class 7 (red) includes 73 locations (i.e., $23 \%$ ) in the Mediterranean area and on the Atlantic coast. 

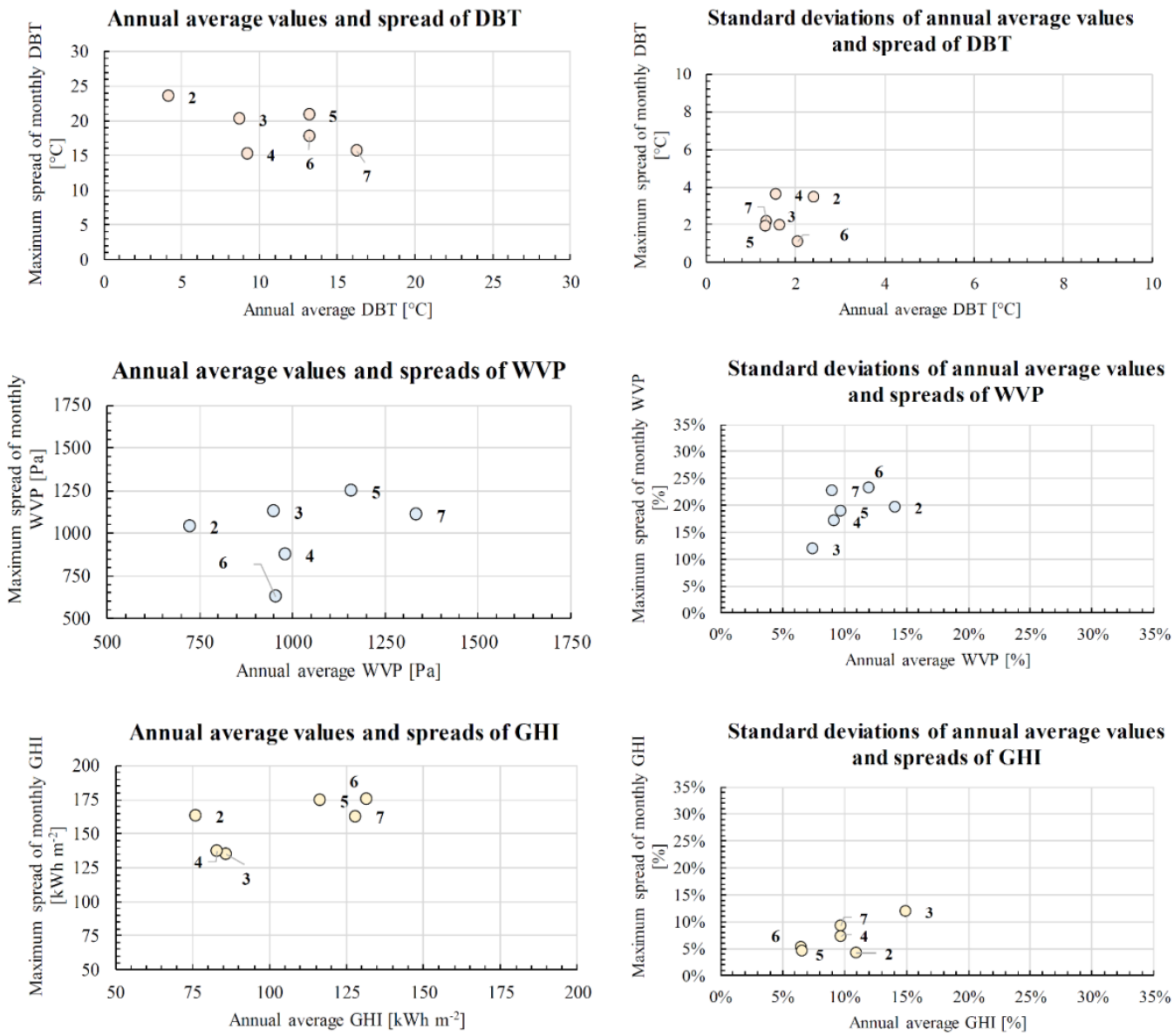

Figure 6: Mean values (left) and standard deviations (right) of annual averages and spreads of dry bulb temperature $(D B T)$, water vapour partial pressure $(W V P)$ and global horizontal irradiation (GHI) for the global clustering.
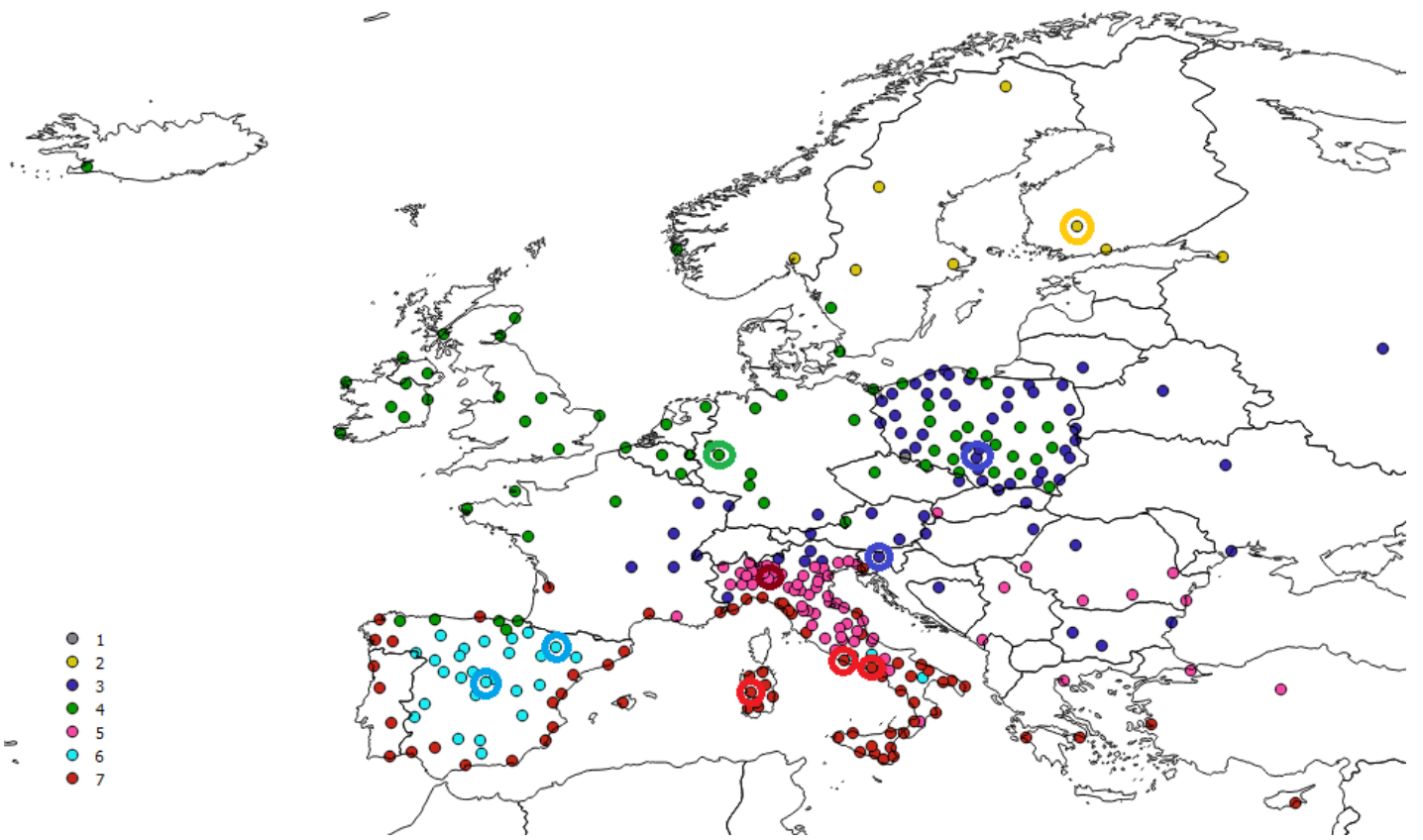

Figure 7: Distribution of the dataset of climates into the 7 classes. The localities with a circle represent the reference climates.

As it can be observed, even if no geographical coordinates were included in clustering, the classes' distribution show in many cases land continuity and a certain degree of consistency with the Köppen-Geiger model.
Identification of representative climates

The procedure described in the methods was employed to identify the representative cities in each climate class. First, the Kolmogorov-Smirnov test was used to see if 
there is statistically significance for some localities (i.e., if, for a given weather variable, the locality is remarkably different from the rest of the class). No significance with respect to a level of $5 \%$ was found, confirming that the classification allowed to build groups with homogeneous annual profiles of $D B T, W V P$ and GHI. The most representative cities resulted:

- Class 2: Tampere, Finland;

- Class 3: Ljubljana, Slovenia, or Czestochowa, Poland;

- Class 4: Koln, Germany;

- Class 5: Milan, Lodi or Varese, Italy (the latter two close to Milan);

- Class 6: Madrid or Huesca, Spain;

- Class 7: Caserta or Latina (between Rome and Naples, Italy) or Oristano, Sardinia.

\section{Conclusion}

In this work we dealt with the topic of climate classification and representative climate identification. First, we discussed the suitability of one of the most popular system, i.e., the Köppen-Geiger classification, for Building Energy Simulation analyses. The KöppenGeiger classes, indeed, are defined basing only on temperature and precipitation data and do not take into account of the effects of other weather variables which play a prominent role in the building energy balance, i.e., the ambient humidity and the solar irradiation. Due to this fact, Köppen-Geiger classes can present partial overlapping issues and insufficient homogeneity to facilitate the identification of a representative climate for each class. To overcome this problem, we proposed the adoption of two statistical techniques, i.e., the Hierarchical Clustering and the Kolmogorov-Smirnov test. Specifically, the clustering and the reference climate identification consider not only annual statistics of temperature, humidity and solar irradiance but also their monthly distributions along the year, allowing to distinguish those localities characterized by similar averages but different seasonal variability and, thus, ensuring more homogeneous classes and higher representativeness of selected climates. The methodology was applied to a dataset of more than 300 European Reference Years. The main findings can be summarized as:

- The grouping of the climates of the analysed dataset into Köppen-Geiger classes showed overlapping issues and poor homogeneity for some of them, suggesting potential for improvement. This was highlighted through both mean values and standard deviations of both annual averages and spreads of dry bulb temperature, water vapour partial pressure and global horizontal irradiation.

- The hierarchical clustering for each variable at a time was able to improve classes separation and homogeneity but just for the considered variable, without a significant impact on the other ones.

- Differently from the procedure adopted in previous contributions (Pernigotto and Gasparella, 2018), no priority of weather variable and consequent subclustering were adopted, opting for a global clustering with all variables included with the same weight. Results showed a general improvement with respect to the previous Köppen-Geiger classification.

- Finally, representative localities were selected based on the results of Kolmogorov-Smirnov tests. In some cases, equivalency was found among more cities, which are found to be either in the same geographical area (e.g., classes 5-7) or representative of separate areas distinguishable within a class (e.g., class $\mathbf{3}$ ).

As a whole, the proposed methodology is expected to provide an analytical reference for climate zoning and for the selection of representative climates, to characterize the building performance across different climatic conditions, supporting the definition of energy policies at regional or international level. Future studies will investigate the application of these results using building performance simulation, as well as the impact of weather data quality and representativeness on the climate clustering.

\section{Acknowledgement}

Funded by the project "Klimahouse and Energy Production", in the framework of the programmaticfinancial agreement with the Autonomous Province of Bozen-Bolzano of Research Capacity Building.

\section{References}

ASHRAE. (2013). ANSI / ASHRAE Standard 169-2013, Climatic Data for Building Design.

Peel, M.C., B.L. Finlayson and T.A. McMahon (2007). Updated world map of the Köppen-Geiger climate classification. Hydrology and Earth System Sciences 11, 1633-1644.

Pernigotto, G., A. Prada, A. Gasparella A. and J.L.M. Hensen (2014). Analysis and improvement of the representativeness of EN ISO 15927-4 reference years for building energy simulation. Journal of Building Performance Simulation 7(6), 391-410.

Pernigotto, G. and A. Gasparella (2018). Classification of European Climates for Building Energy Simulation Analyses. Proceedings of the V International High Performance Buildings Conference at Purdue. West Lafayette (Indiana, U.S.), 9-12 July 2018.

Walsh, A., D. Cóstola and L.C. Labaki (2017). Review of methods for climatic zoning for building energy efficiency programs. Building and Environment 112, 337-350.

Walsh, A., D. Cóstola and L.C. Labaki (2018). Performance-based validation of climatic zoning for building energy efficiency applications, Applied Energy 212, 416-427. 